\section{Evolutionary Computation for Dynamic Optimization Problems}

\section{Shengxiang Yang}

Centre for Computational Intelligence

School of Computer Science and Informatics

De Montfort University, United Kingdom

$$
\text { Email: syang@dmu.ac.uk }
$$

http://www.tech.dmu.ac.uk/ syang

http://www.sigevo.org/gecco-2013/ .
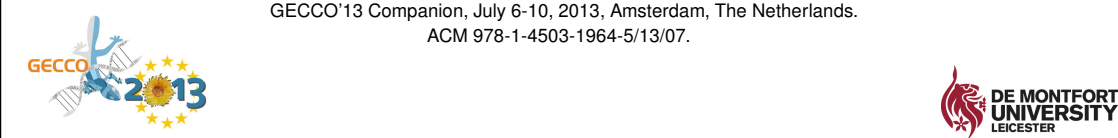

Shengxiang Yang

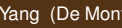

Universi
Centre for CI (CCl), De Montfort University

- $\mathrm{CCl}$ (www.cci.dmu.ac.uk):

- Mission: Developing fundamental theoretical and practical solutions to real world problems using a variety of $\mathrm{Cl}$ paradigms

- Members: 15 staff, 4 research fellows, 30+ PhDs, visiting researchers

- Components: 3 Labs (Robotics, Gaze, Game Programming) \& Bio-Health Informatics Research Group

- Funding:

- Research Councils: EPSRC, TSB, Royal Academy of Engineering, Royal Society, KTP, Innovation Fellowships, HOPE foundation

- Government: Leicester City Council, DTI

- Industries: Lachesis, EMDA, RSSB, Network Rail, etc.

- Collaborations:

- Universities: UK, USA, Spain, and China

- Industries and local governments

- Teaching/Training:

- DTP-IS: University Doctor Training Programme in Intelligent Systems

- MSc Intelligent Systems, MSc Intelligent Systems \& Robotics

- BSc Artificial Intelligence with Robotics

- YouTube page: http://www.youtube.com/thecci

\section{Instructor/Presenter — Shengxiang Yang}

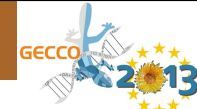

- Education and career history:

- PhD, Northeastern University, China, 1999

- Worked at King's College London, University of Leicester, and Brunel University, 1999-2012

- Joined De Montfort University as Professor in Computational Intelligence (CI) in July 2012

- Director of Centre for Computational Intelligence (CCI)

- Research interests:

- Evolutionary computation (EC) and nature-inspired computation

- Dynamic optimisation and multi-objective optimisation

- Relevant real-world applications

- Over 160 publications and over £1M funding as the PI

- Editor, Evolutionary Computation and 3 other journals

- Chair of two IEEE CIS Task Forces

- EC in Dynamic and Uncertain Environments

- Intelligent Network Systems

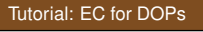

\section{Outline of the Tutorial}




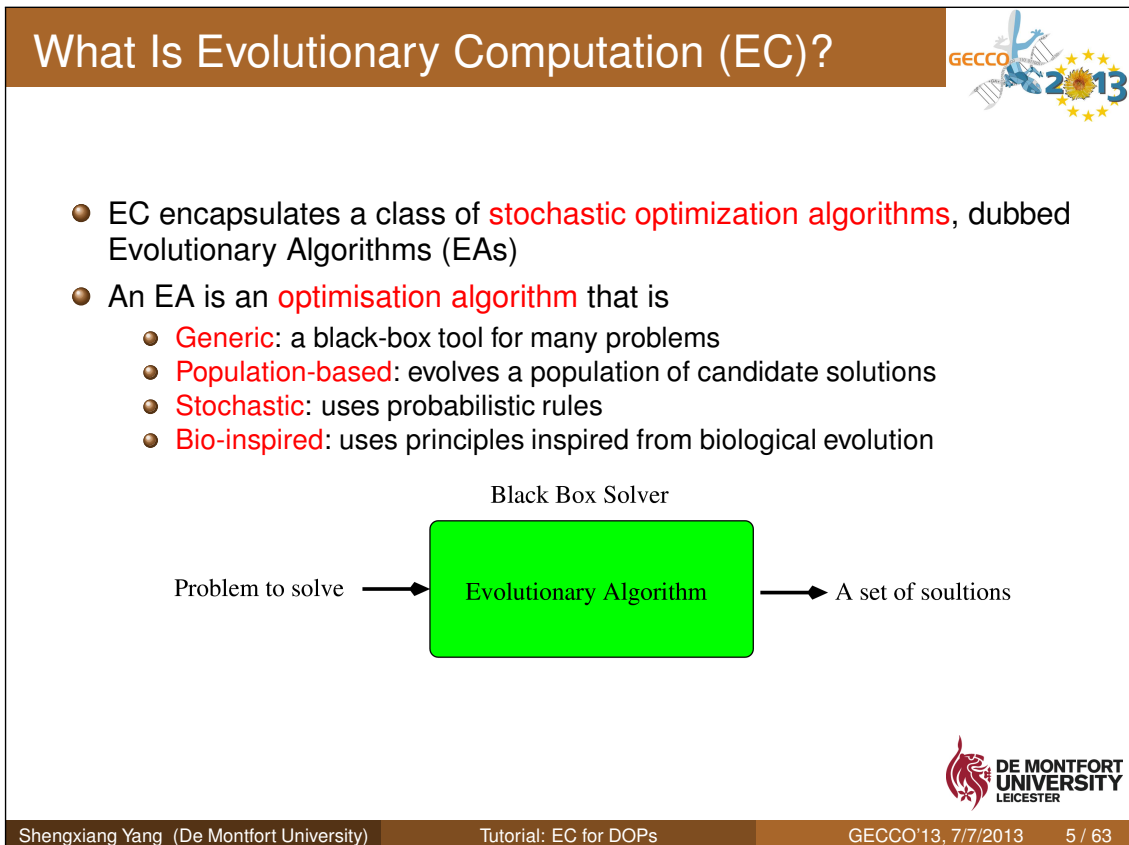

EC Applications

- EAs are easy-to-use: No strict requirements to problems

- Widely used for optimisation and search problems

- Financial and economical systems

- Transportation and logistics systems

- Industry engineering

- Automatic programming, art and music design

- .......

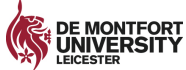

Sengxiang

(De

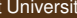

Tutorial: EC for DOPs

\section{EC for Optimisation Problems}

\section{Design and Framework of an EA}

Given a problem to solve, first consider two key things:

- Representation of solution into individual

- Evaluation or fitness function

Then, design the framework of an EA:

- Initialization of population

- Evolve the population

- Selection of parents

- Variation operators (recombination \& mutation)

- Selection of offspring into next generation

- Termination condition: a given number of generations

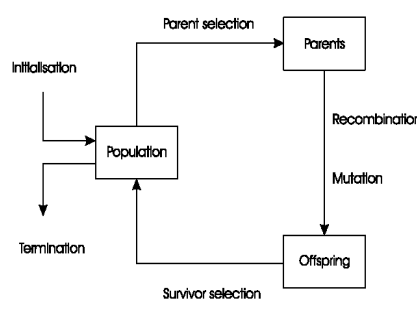

(द) UNIVNTEORT

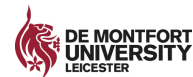

Traditionally, research on EAs has focused on static problems

- Aim to find the optimum quickly and precisely

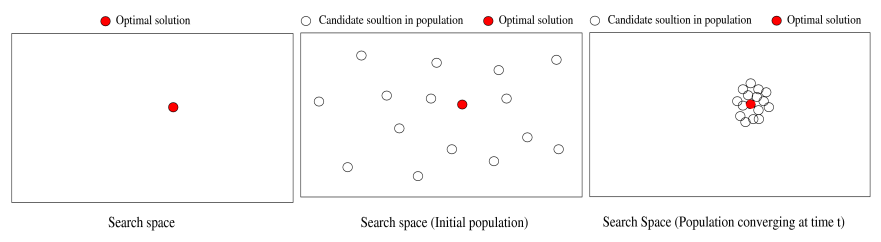

- But, many real-world problems are dynamic optimization problems (DOPs), where changes occur over time

- In transport networks, travel time between nodes may change
- In logistics, customer demands may change

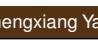

ng (De 


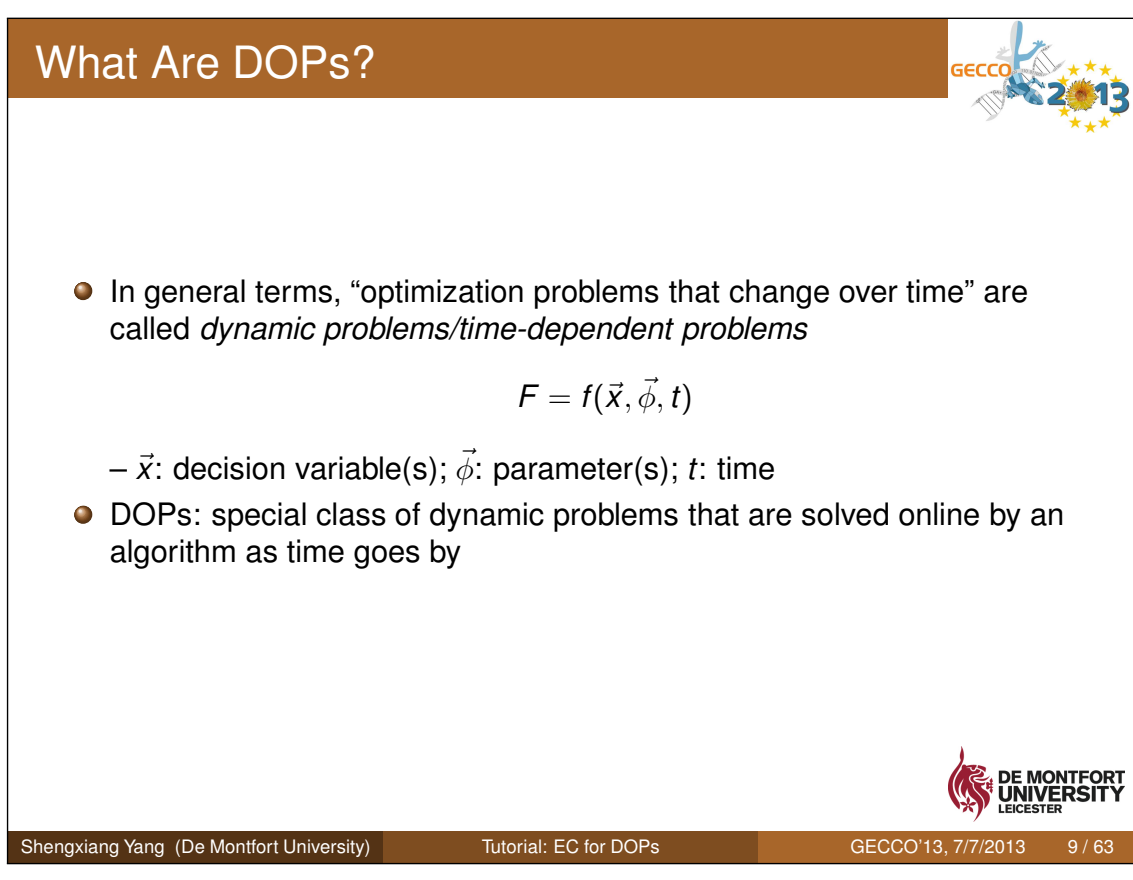

\section{Why DOPs Challenge EC?}

- For DOPs, optima may move over time in the search space

- Challenge: need to track the moving optima over time

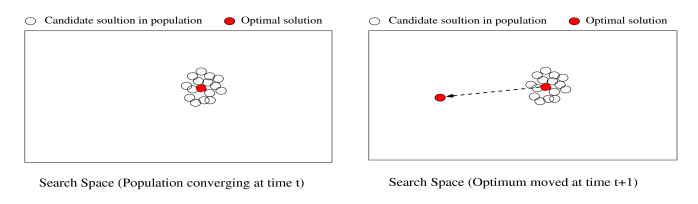

- DOPs challenge traditional EAs

- Once converged, hard to escape from an old optimum
Why EC for DOPs?

- Many real-world problems are DOPs

- EAs, once properly enhanced, are good choice

- Inspired by natural/biological evolution, always in dynamic environments

- Intrinsically, should be fine to deal with DOPs

- Many events on EC for DOPs recently

\section{Relevant Events}

- Books (Monograph or Edited):

- Yang \& Yao, 2013; Yang et al., 2007; Morrison, 2004; Weicker, 2003; Branke, 2002

- PhD Theses:

- Mavrovouniotis, 2013; du Plessis, 2012; Li, 2011; Nguyen, 2011; Simoes, 2010

- Journal special issues:

- Neri \& Yang, 2010; Yang et al., 2006; Jin \& Branke, 2006; Branke, 2005

- Workshops and conference special sessions:

- EvoSTOC (2004-2013): part of Evo*

- ECiDUE (2004-2013): part of IEEE CEC

- EvoDOP ('99, '01, '03, '05, '07, '09): part of GECCO

- IEEE Symposium on CIDUE (Paris, 2011; Singapore, 2013)

- IEEE Competitions: within IEEE CEC 2009 \& CEC 2012 


\section{Benchmark and Test DOPs}

- Basic idea: change base static problem(s) to create DOPs

- Real space:

- Switch between different functions

- Move/reshape peaks in the fitness landscape

- Binary space:

- Switch between $\geq 2$ states of a problem: knapsack

- Use binary masks: XOR DOP generator (Yang \& Yao'05)

- Combinatorial space:

- Change decision variables: item weights/profits in knapsack problems

- Add/delete decision variables: new jobs in scheduling, nodes added/deleted in network routing problems

\section{The DF1 Generator}

- Proposed by Morrison \& De Jong (1999)

- The base landscape in the $D$-dimensional real space:

$$
f(\vec{x})=\max _{i=1, \ldots, p}\left[H_{i}-R_{i} \times \sqrt{\sum_{j=1}^{D}\left(x_{j}-X_{i j}\right)^{2}}\right]
$$

$-\vec{x}=\left(x_{1}, \cdots, x_{D}\right)$ : a point in the landscape; $p$ : number of peaks

$-H_{i}, R_{i}, X_{i}=\left(X_{i 1}, \cdots, X_{i D}\right)$ : height, slope, center of peak $i$

- The dynamics is controlled by a logistics function:

$$
\Delta_{t}=A \cdot \Delta_{t-1} \cdot\left(1-\Delta_{t-1}\right)
$$

$-A \in[1.0,4.0]:$ a constant; $\Delta_{t}$ : step size of changing a parameter

\section{Moving Peaks Benchmark (MPB) Problem}

- Proposed by Branke (1999)

- The MPB problem in the $D$-dimensional space:

$$
F(\vec{x}, t)=\max _{i=1, \ldots, p} \frac{H_{i}(t)}{1+W_{i}(t) \sum_{j=1}^{D}\left(x_{j}(t)-X_{i j}(t)\right)^{2}}
$$

$-W_{i}(t), H_{i}(t), X_{i}(t)=\left\{X_{i 1} \cdots X_{i D}\right\}:$ height, width, location of peak $i$ at $t$

- The dynamics:

$$
\begin{gathered}
H_{i}(t)=H_{i}(t-1)+\text { height_severity } * \sigma \\
W_{i}(t)=W_{i}(t-1)+\text { width_severity } * \sigma \\
\vec{v}_{i}(t)=\frac{s}{\left|\vec{r}+\vec{v}_{i}(t-1)\right|}\left((1-\lambda) \vec{r}+\lambda \vec{v}_{i}(t-1)\right) \\
\vec{X}_{i}(t)=\vec{X}_{i}(t)(t-1)+\vec{v}_{i}(t)
\end{gathered}
$$

$-\sigma \sim N(0,1) ; \lambda$ : correlated parameter

- $\vec{v}_{i}(t)$ : shift vector, which combines random vector $\vec{r}$ and $\vec{v}_{i}(t+1)$ and is normalized to the shift length $s$

\section{Tutorial: EC for DOPs}

\section{GECCO'13,}

\section{Dynamic Knapsack Problems (DKPs)}

- Static knapsack problem:

- Given $n$ items, each with a weight and a profit, and a knapsack with a fixed capacity, select items to fill up the knapsack to maximize the profit while satisfying the knapsack capacity constraint

- The DKP:

- Constructed by changing weights and profits of items, and/or knapsack capacity over time as:

$$
\operatorname{Max} f(\vec{x}(t), t)=\sum_{i=1}^{n} p_{i}(t) \cdot x_{i}(t), \text { s.t. }: \sum_{i=1}^{n} w_{i}(t) \cdot x_{i}(t) \leq C(t)
$$

$-\vec{x}(t) \in\{0,1\}^{n}:$ a solution at time $t$

$-x_{i}(t) \in\{0,1\}:$ indicates whether item $i$ is included or not

$-p_{i}(t)$ and $w_{i}(t)$ : profit and weight of item $i$ at $t$

$-C(t)$ : knapsack capacity at $t$ 


\section{The XOR DOP Generator}

- The XOR DOP generator can create DOPs from any binary $f(\vec{x})$ by an XOR operator " $\oplus$ " (Yang, 2003; Yang \& Yao, 2005)

- Suppose the environment changes every $\tau$ generations

- For each environmental period $k=\lfloor t / \tau\rfloor$, do:

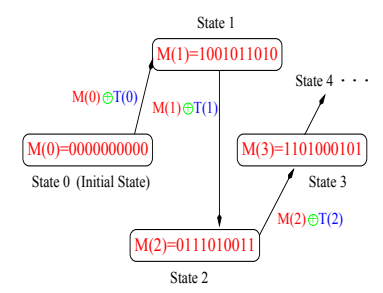

(1) Create a template $T_{k}$ with $\rho * I$ ones

(2) Create a mask $\vec{M}(k)$ incrementally $\vec{M}(0)=\overrightarrow{0}$ (the initial state) $\vec{M}(k+1)=\vec{M}(k) \oplus \vec{T}(k)$

Evaluate an individual:

$$
f(\vec{x}, t)=f(\vec{x} \oplus \vec{M}(k))
$$

$T(0)=100101010 \quad T(1)=11000001 \quad T(2)=100001010$

- $\tau$ and $\rho$ controls the speed and severity of change respectively

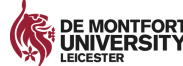

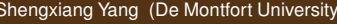

\section{Constructing Cyclic Dynamic Environments}

Can extend the XOR DOP generator to create cyclic environments:

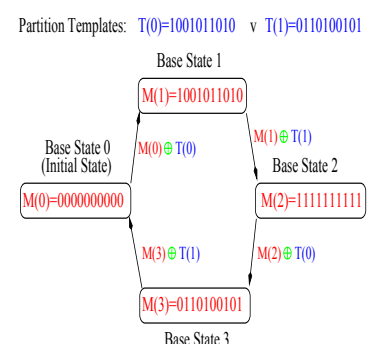

- Construct $K$ templates $\vec{T}(0), \cdots, \vec{T}(K-1)$

- Form a partition of the search space

- Each contains $\rho \times I=I / K$ ones

(2) Create $2 K$ masks $\vec{M}(i)$ as base states

$$
\vec{M}(0)=\overrightarrow{0} \text { (the initial state) }
$$

$\vec{M}(i+1)=\vec{M}(i) \oplus \vec{T}(i \% K), i=0, \cdots, 2 K-1$

(3) Cycle among $\vec{M}(i)$ 's every $\tau$ generations

$$
f(\vec{x}, t)=f\left(\vec{x} \oplus \vec{M}\left(I_{t}\right)\right)=f(\vec{x} \oplus \vec{M}(k \%(2 K)))
$$

$-k=\lfloor t / \tau\rfloor:$ environmental index

$-I_{t}=k \%(2 K):$ mask index

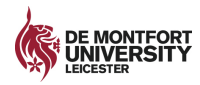

\section{Constructing Cyclic Environments with Noise}

We can also construct cyclic environments with noise:

- Each time before a base state is entered, it is bitwise changed with a small probability

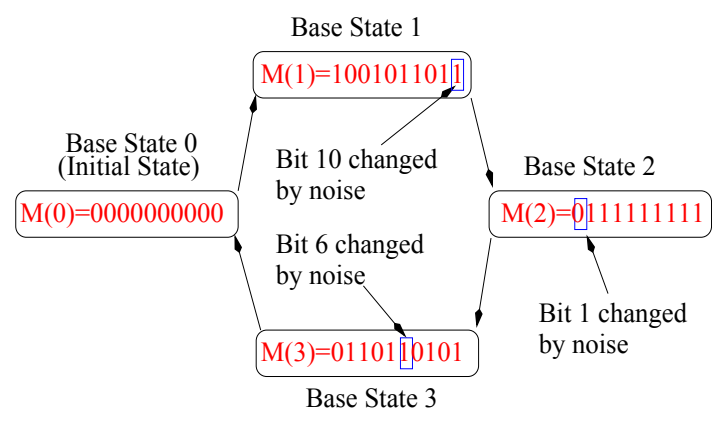

(4.) DENONTEORT
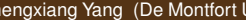

\section{Dynamic Traveling Salesman Problems}

- Stationary traveling salesman problem (TSP):

- Given a set of cities, find the shortest route that visits each city once and only once

- Dynamic TSP (DTSP):

- May involve dynamic cost (distance) matrix

$$
D(t)=\left\{d_{i j}(t)\right\}_{n * n}
$$

$-d_{i j}(t)$ : cost from city $i$ to $j ; n$ : the number of cities

- The aim is to find a minimum-cost route containing all cities at time $t$

- DTSP can be defined as $f(x, t)$ :

$$
f(x, t)=\operatorname{Min}\left(\sum_{i=1}^{n} d_{x_{i}, x_{i+1}}(t)\right)
$$

where $x_{i} \in 1, \cdots, n$. If $i \neq j, x_{i} \neq x_{j}$, and $x_{n+1}=x_{1}$ 


\section{Dynamic Permutation Benchmark Generator}

- The dynamic benchmark generator for permutation-encoded problems (DBGP) can create a DOP from any stationary TSP/VRP by swapping objects:

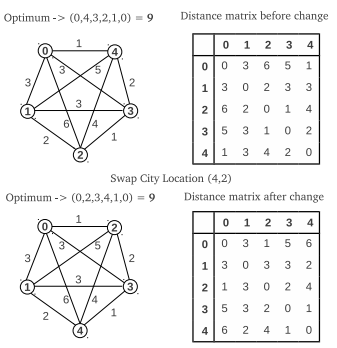

(1) Generate a random vector $\vec{r}(T)$ that contains all objects every $f$ iterations

(2) Generate another randomly re-order vector $\overrightarrow{r^{\prime}}(T)$ that contains only the first $m \times n$ objects of $\vec{r}(T)$

(3) Modify the encoding of the problem instance with $m \times n$ pairwise swaps

- More details: M. Mavrovouniotis, S. Yang, \& X. Yao (2012). PPSN XII, Part II, LNCS 7492, pp. 508-517

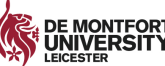
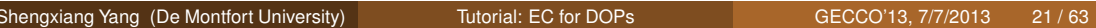

\section{Tutorial: EC for DOPs}

\section{Effect on Algorithms}

- Similar with the XOR DOP generator, DBGP shifts the population of an alg. to new location in the fitness landscape

- The individual with the same encoding as before a change will have a different cost after the change

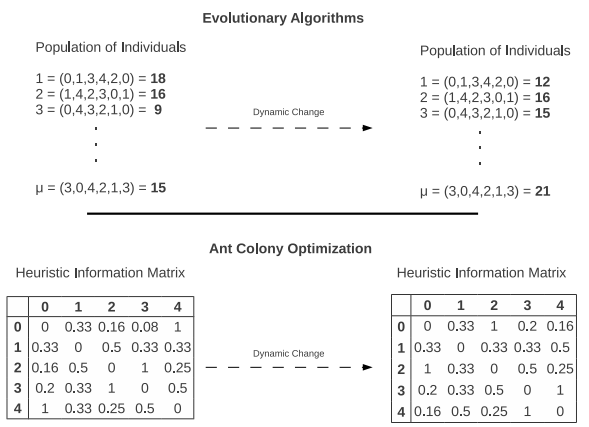

- Can extend for cyclic and cyclic with noise environments
Generalized DOP Benchmark Generator (GDBG)

- Proposed by Li \& Yang (2008), GDBG uses the model below:

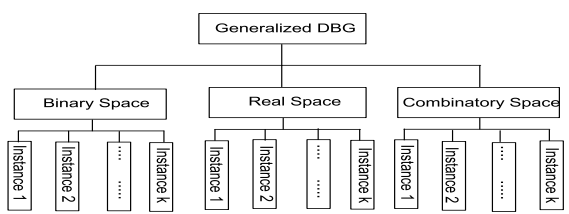

- In GDBG, DOPs are defined as:

$$
F=f(x, \phi, t),
$$

- $\phi$ : system control parameter

- Dynamism results from tuning $\phi$ of the current environment

$$
\phi(t+1)=\phi(t) \oplus \Delta \phi
$$

$-\Delta \phi$ : deviation from the current control parameter(s)

- The new environment at $t+1$ is as follows:

$$
f(x, \phi, t+1)=f(x, \phi(t) \oplus \Delta \phi, t)
$$
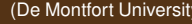

\section{GDBG: Dynamic Change Types}

- Change types:

(C) Small step: $\Delta \phi=\alpha \cdot\|\phi\| \cdot \operatorname{rand}()$

(2) Large step: $\Delta \phi=\|\phi\| \cdot(\alpha+(1-\alpha) \operatorname{rand}())$

(3) Random: $\Delta \phi=\|\phi\| \cdot \operatorname{rand}()$

(4) Chaotic: $\phi(t+1)=A \cdot \phi(t) \cdot(1-\phi(t) /\|\phi\|)$

(5) Recurrent: $\phi(t+1)=\phi(t \% P)$

6 Recurrent with nosy: $\phi(t+1)=\phi(t \% P)+\alpha \cdot\|\phi\| \cdot \operatorname{rand}()$

(7) .......

- More details:

- C. Li \& S. Yang (2008). SEAL'08, LNCS 5361, pp. 391-400

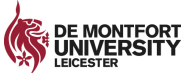




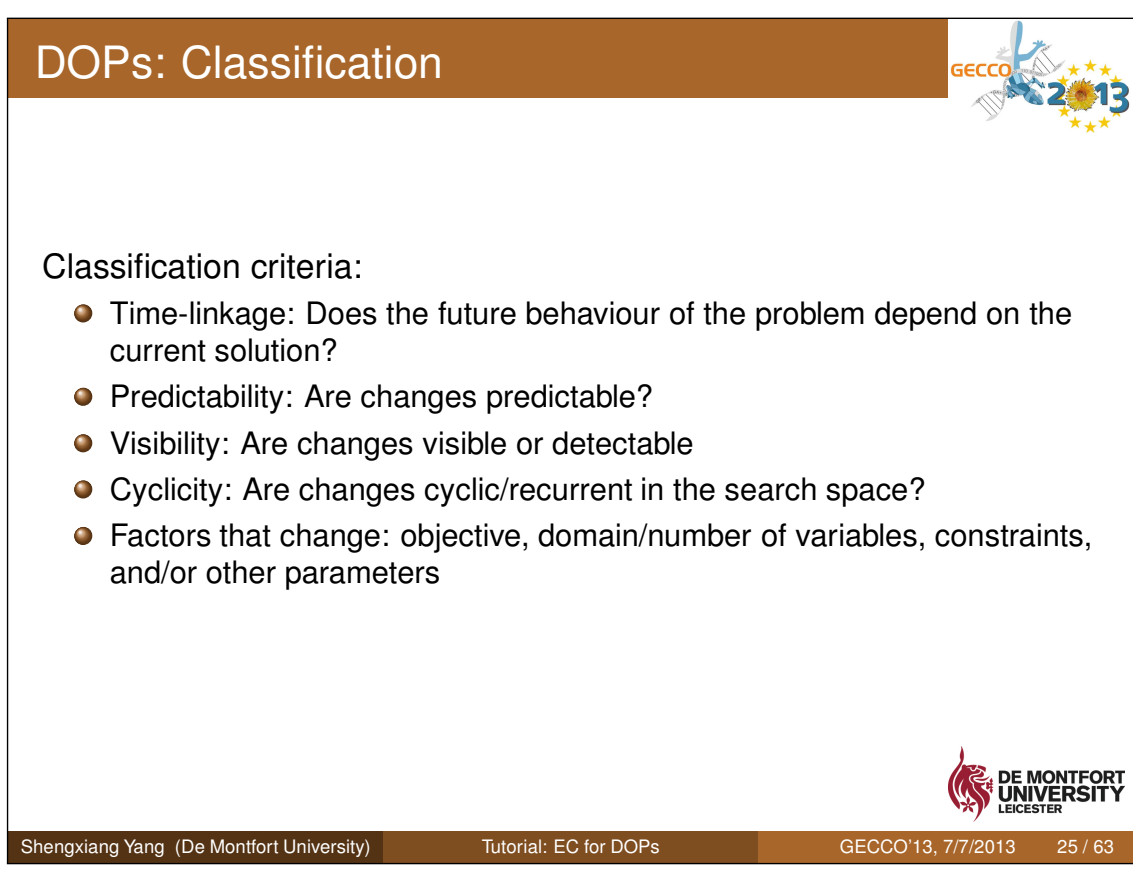

\section{DOPs: Common Characteristics}

Common characteristics of DOPs in the literature:

- Most DOPs are non time-linkage problems

- For most DOPs, changes are assumed to be detectable

- In most cases, the objective function is changed

- Many DOPs have unpredictable changes

- Most DOPs have cyclic/recurrent changes

\section{Performance Measures}

- For EC for stationary problems, 2 key performance measures

- Convergence speed

- Success rate of reaching optimality

- For EC for DOPs, over 20 measures (Nguyen et al., 2012)

- Optimality-based performance measures

- Collective mean fitness or mean best-of-generation

- Accuracy

- Offline error and offline performance

- Mean distance to optimum at each generation

- .......

- Behaviour-based performance measures

- Reactivity

- Stability

- Robustness

- Satisficability

- Diversity measures

- .......

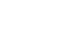

Montfort

\section{Performance Measures: Examples}

- Collective mean fitness (mean best-of-generation):

$$
\bar{F}_{B O G}=\frac{1}{G} \times \sum_{i=1}^{i=G}\left(\frac{1}{N} \times \sum_{j=1}^{j=N} F_{B O G_{i j}}\right)
$$

$-G$ and $N$ : number of generations and runs, resp.

$-F_{B O G_{i j}}$ : best-of-generation fitness of generation $i$ of run $j$

- Adaptation performance (Mori et al., 1997)

$$
\operatorname{Ada}=\frac{1}{T} \sum_{t=1 . . T}\left(f_{\text {best }}(t) / f_{\text {opt }}(t)\right)
$$

- Accuracy (Trojanowski and Michalewicz, 1999)

$$
A c c=\frac{1}{K} \sum_{i=1 . . K}\left(f_{\text {best }}(i)-f_{\text {opt }}(i)\right)
$$

$-f_{\text {best }}(i)$ : best fitness for environment $i$ (best before change) 


\section{Part II: Play the Game}

- EC approaches for DOPs

- Case studies

- Relevant issues

- Future work
EC for DOPs: General Approaches

- Many approaches developed to enhance EAs for DOPs

- Typical approaches:

- Memory: store and reuse useful information

- Diversity: handle convergence directly

- Multi-population: co-operate sub-populations

Adaptive: adapt generators and parameters

- Prediction: predict changes and take actions in advance

- They have been applied to different EAs for DOPs

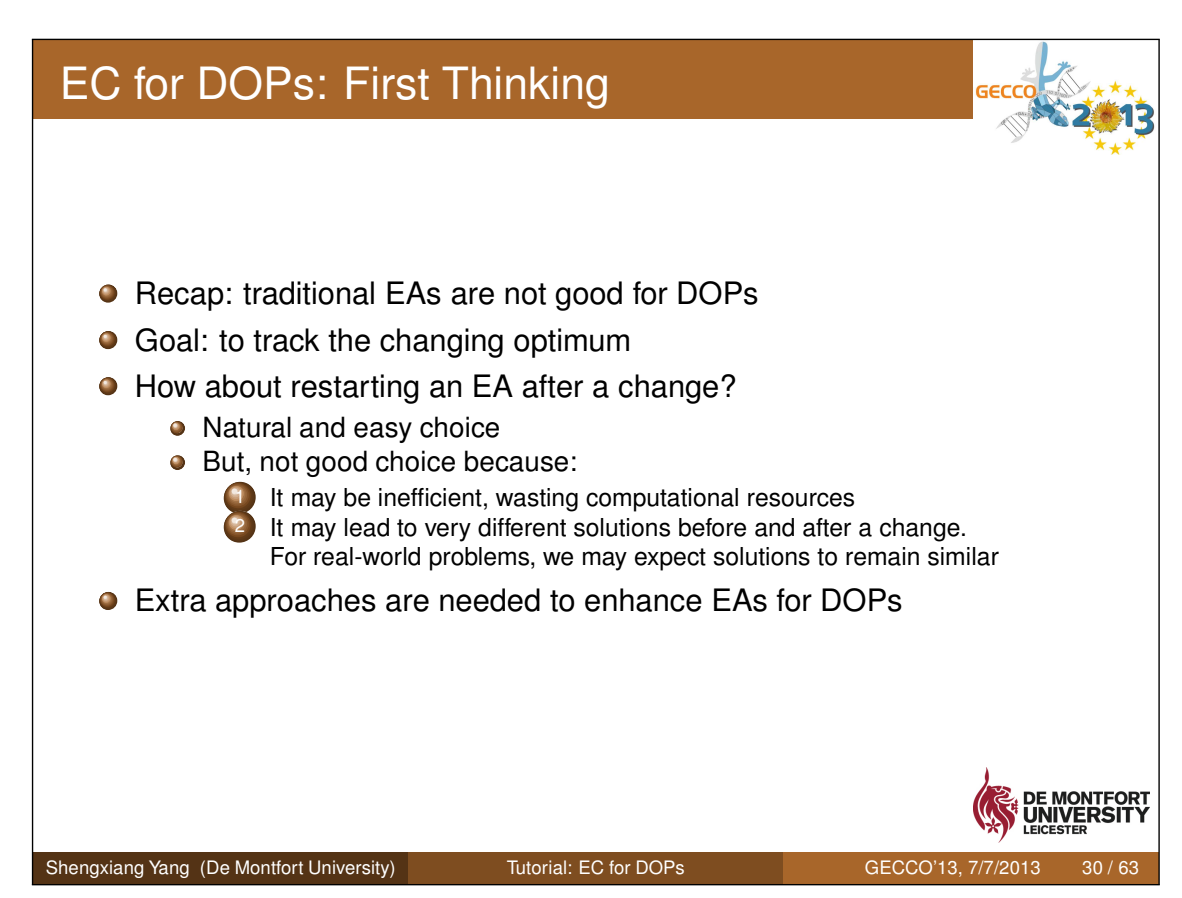

\section{Memory Approaches}

- Cyclic DOPs: change cyclically among a fixed set of states

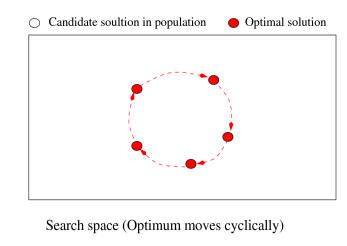

- Memory works by storing and reusing useful information

- Two classes regarding how to store information

- Implicit memory: uses redundant representations

- Multiploidy and dominance (Ng \& Wong, 1995; Lewis et al., 1998)

- Dualism mechanisms (Yang, 2003; Yang \& Yao, 2005)

- Explicit memory: uses extra space to store information 


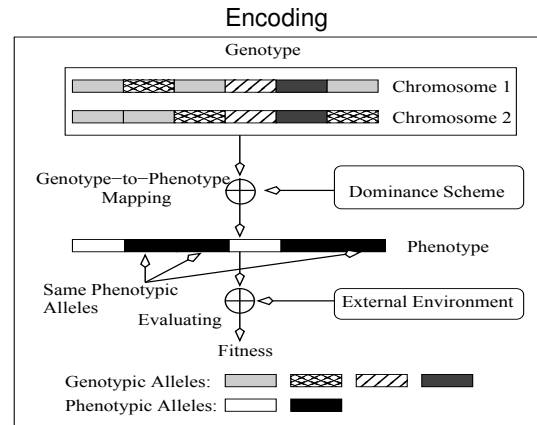

Dominance Scheme

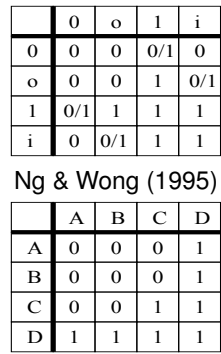

Lewis et al. (1998)

- Each individual has a pair of chromosomes

- Dominance scheme maps genotype to phenotype

- Dominance scheme may change or be adaptive (Uyar \& Harmanci, 2005)

\section{Explicit Memory Approaches}

\section{Basic idea: use extra memory}

- With time, store useful information of the pop into memory

- When a change occurs, use memory to track new optimum

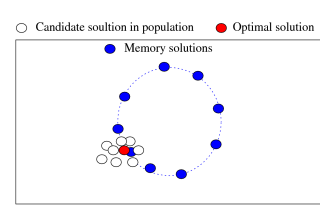

Search space (Memory stores best solutions)

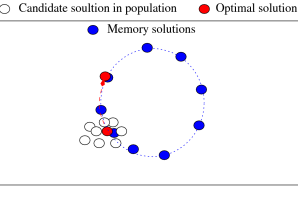

Search space (Opimum moves to next state)

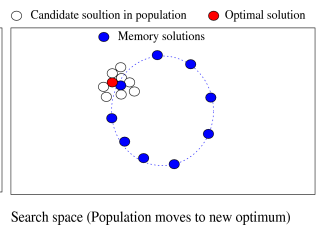

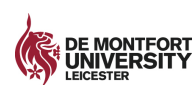

\section{Explicit Memory: Direct vs Associative}

- Direct memory: store good solutions (Branke, 1999)

- Associative memory: store environmental information + good solutions (Yang \& Yao, 2008)

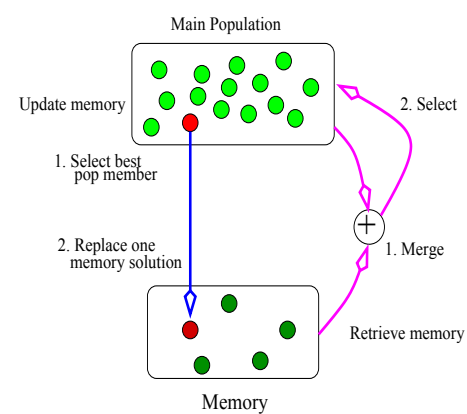

Direct Memory

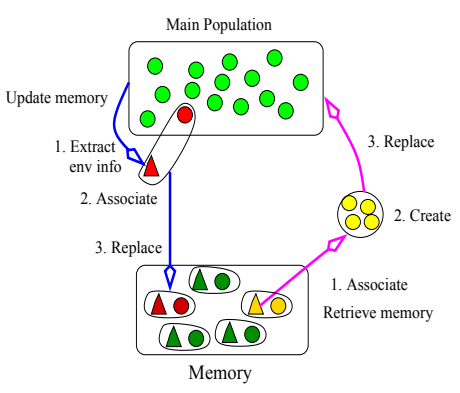

$\triangle$ Environment information $\bigcirc$ Solution

Associative Memory

\section{Associative Memory Based Genetic Algorithm}

Idea: Use allele distribution (AD) $\vec{D}$ to represent environmental info.

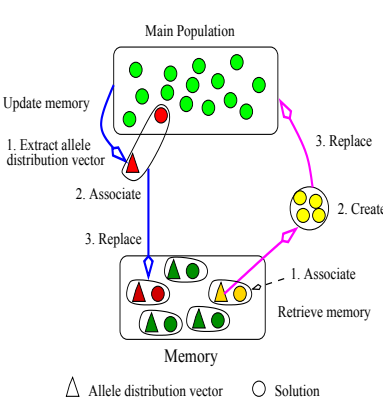

- Use memory to store $<\vec{D}, S>$ pairs

- Update memory by similarity policy

- Re-evaluate memory every generation. If change detected

- Extract best memory AD: $\vec{D}_{M}$

- Create solutions by sampling $\vec{D}_{M}$

- Replace them into the pop randomly

- Details:

- S. Yang (2006). EvoWorkshops'06, pp. 788-799

$\triangle$ Allele distribution vector $\quad O$ Solution 


\section{Diversity Approaches: Random Immigrants}

- Convergence is the key problem in metaheuristics for DOPs

- Random immigrants:

- Each generation, insert some random individuals (called random immigrants) into the population to maintain diversity

- When optimum moves, random immigrants nearby take action to draw the pop to the new optimum
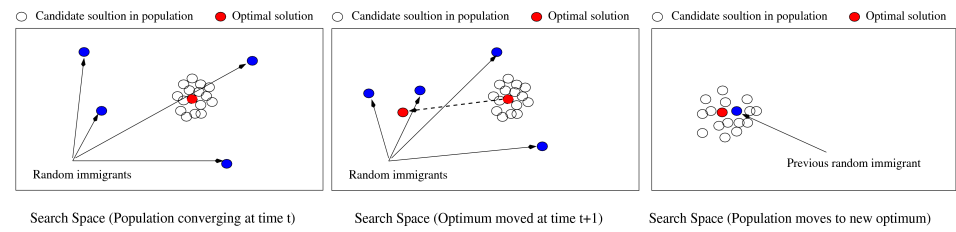

Search Space (Population converging a t time t)

Search Space (Optimum moved at time t+1) Search Space (Popplation moves to new optimum)

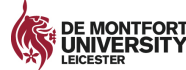

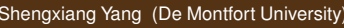

Tutorial: EC for DOP

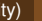

\section{Memory-Based Immigrants}

- Random immigrants maintain the diversity while memory adapts an algorithm directly to new environments

- Memory-based immigrants: uses memory to guide immigrants towards current environment

- Re-evaluate the memory every generation

- Retrieve the best memory point $B_{M}(t)$ as the base

- Generate immigrants by mutating $B_{M}(t)$ with a prob.

- Replace worst members in the population by these immigrants

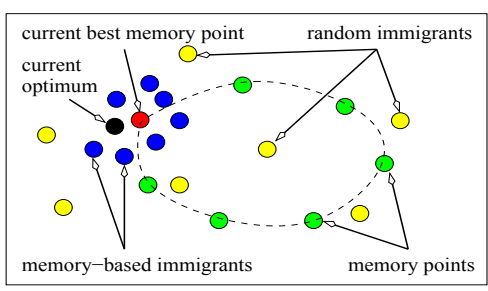

Search Space

\section{Experimental Results: Immigrants Based GAs}
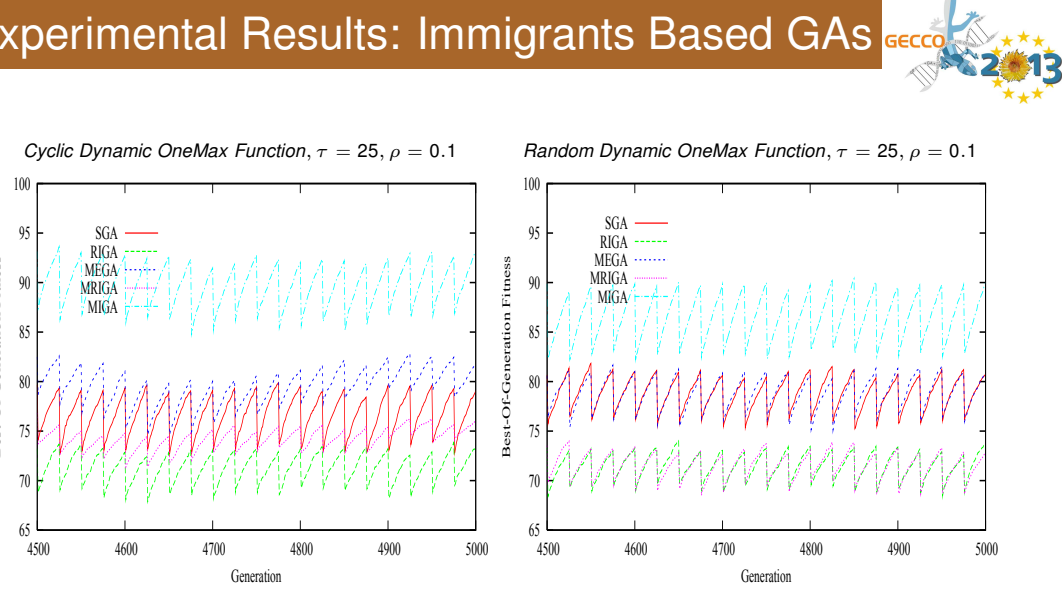

- Memory-based immigrants GA (MIGA) significantly beats other GAs

- More details:

- S. Yang (2008). Evol. Comput., 16(3): 385-416

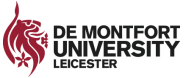

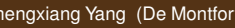

\section{GECCO'13, 77+}

\section{Hybrid Immigrants Approach}

- Combines elitism, dualism and random immigrants ideas

- Dualism: Given $\vec{x}=\left(x_{1}, \cdots, x_{l}\right) \in\{0,1\}^{\prime}$, its dual is defined as

$$
\vec{x}^{d}=\operatorname{dual}(\vec{x})=\left(x_{1}^{d}, \cdots, x_{I}^{d}\right) \in\{0,1\}^{\prime}
$$

where $x_{i}^{d}=1-x_{i}$

- Each generation $t$, select the best individual from previous generation,

$E(t-1)$, to generate immigrants

- Elitism-based immigrants: Generate a set of individuals by mutating $E(t-1)$ to address slight changes

- Dualism-based immigrants: Generate a set of individuals by mutating the dual of $E(t-1)$ to address significant changes

- Random immigrants: Generate a set of random individuals to address medium changes

- Replace these immigrants into the population

- More details:

- S. Yang \& R. Tinos (2007). Int. J. of Autom. \& Comp., 4(3): 243-254 


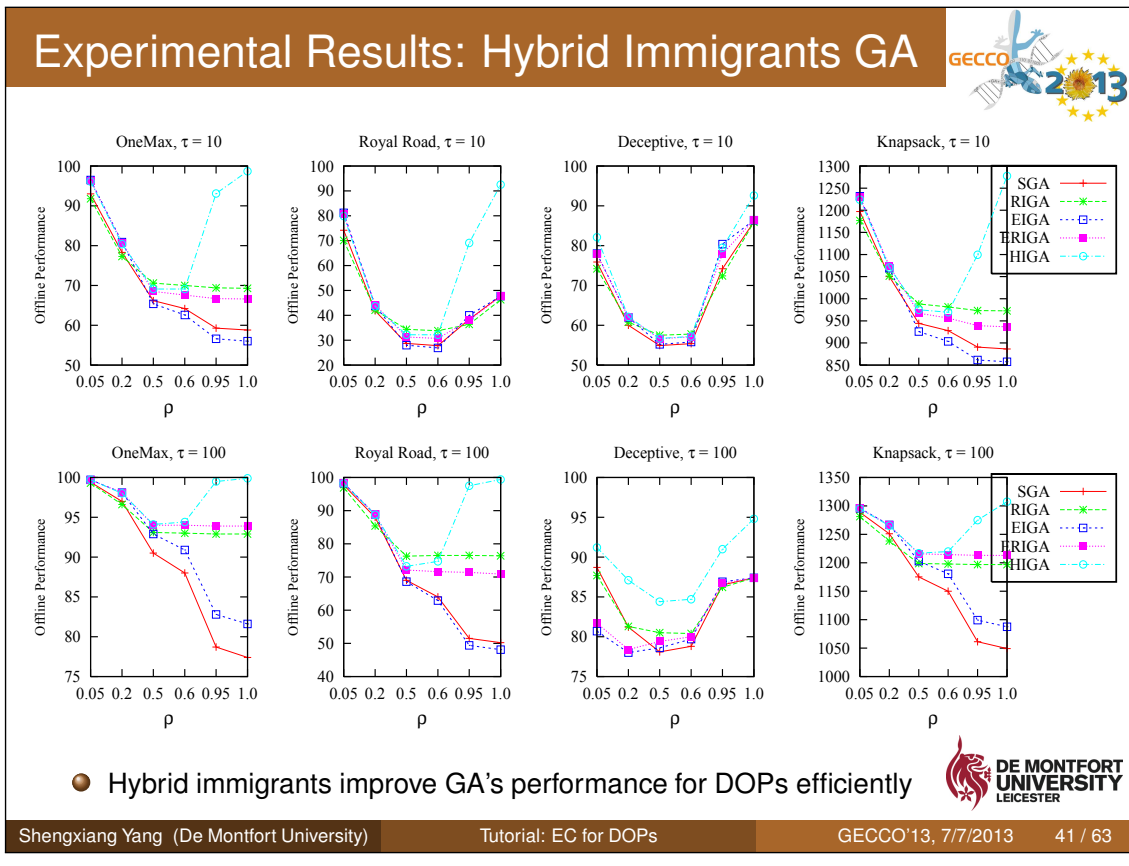

Multi-Populations: Shifting Balance

- Multi-population scheme uses co-operating sub-populations

- Shifting Balance GA (Oppacher \& Wineberg, 1999):

- A core population exploits the promising area

- Several colonies explore the search space

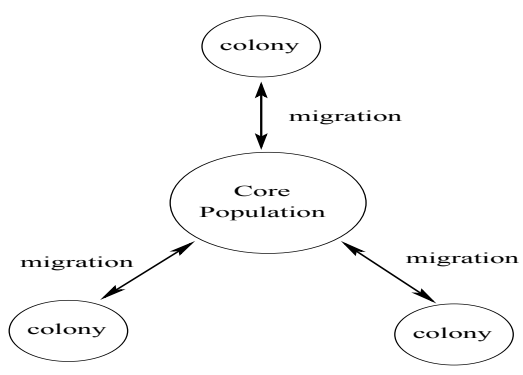

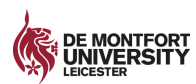

Multi-Populations: Self-Organizing Scouts

- Self-organizing scouts (SOS) GA (Branke et al., 2000)

- The parent population explores the search space

- A child population is split under certain conditions

- Child populations search limited promising areas

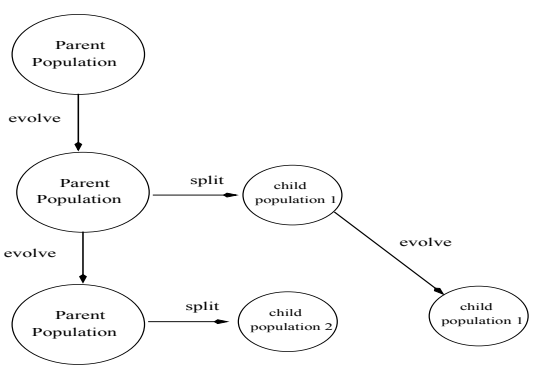

(4)i

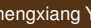

rsity)

Tutorial: EC for DOPs

\section{Multi-Populations: Clustering PSO}

- Particle Swarm Optimisation (PSO):

- Motivated by the social behaviour of swarm of animals, e.g., bird flocking and fish schooling

- PSO has been used to address DOPs

- Recently, we developed a Clustering PSO (CPSO) for DOPs

- Use a clustering technique to construct sub-swarms

- Each sub-swarm will search among one peak quickly

- Overlapping and convergence check

- Strategies to response to changes

- More details:

- S. Yang \& C. Li (2010). IEEE Trans Evol Comput, 14(6): 93-106 


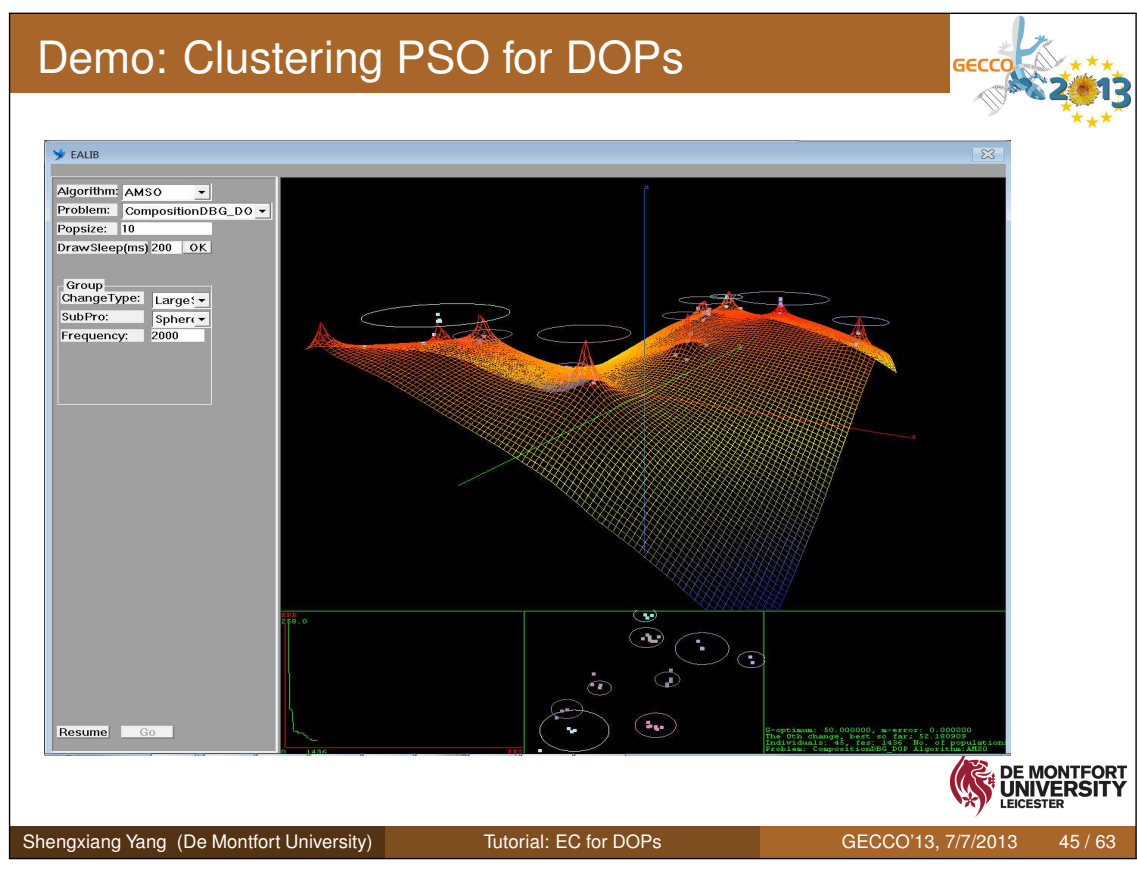

Prediction Approaches

- For some DOPs, changes exhibit predictable patterns

- Techniques (forecasting, Kalman filter, etc.) can be used to predict

- The location of the next optimum after a change

When the next change will occur and which environment may appear

- Some relevant work: see Simões \& Costa (2009)

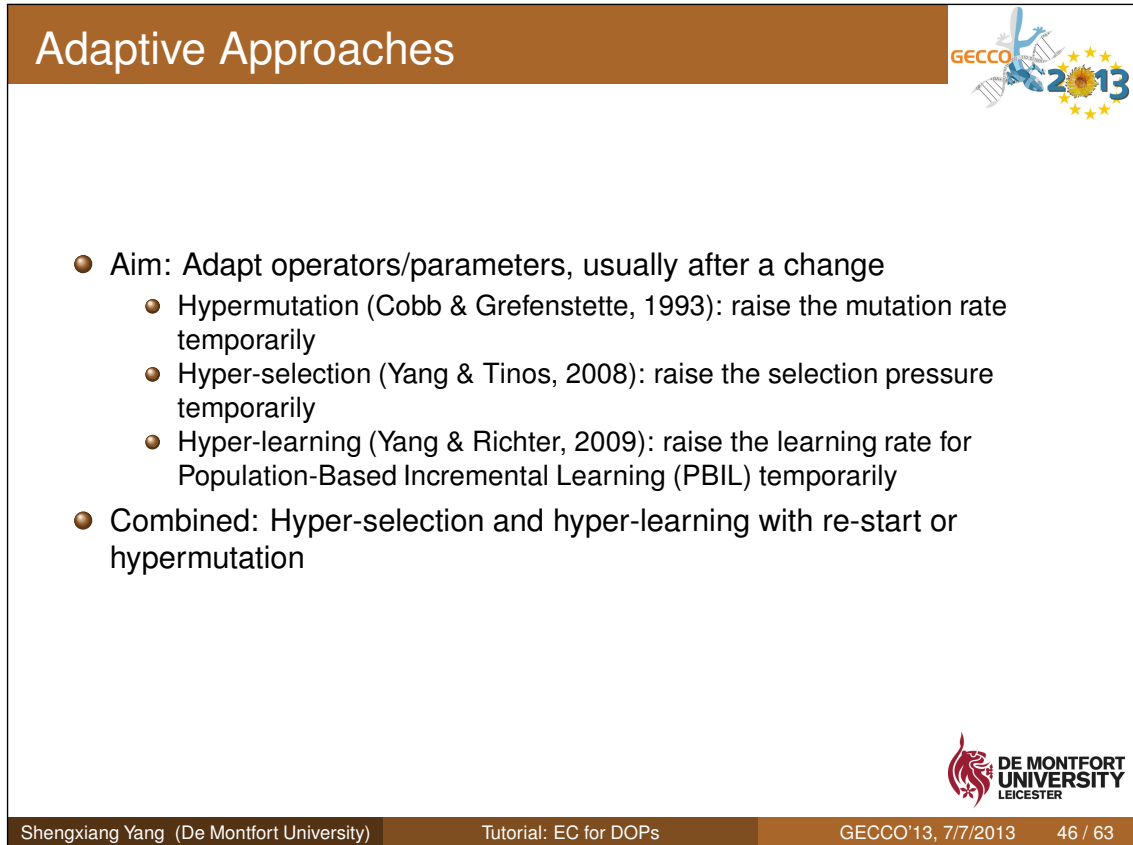

Remarks on Enhancing Approaches

- No clear winner among the approaches

- Memory is efficient for cyclic environments

- Multi-population is good for tracking competing peaks - The search ability will decrease if too many sub-populations

- Diversity schemes are usually useful

- Guided immigrants may be more efficient

- Different interaction exists among the approaches

- Golden rule: balancing exploration \& exploitation over time 
Case Study: Dynamic Routing in MANETs - 1

- Shortest path routing problem (SPRP) in a fixed network:

- Find the shortest path between source and destination in a fixed topology

- More and more mobile ad hoc networks (MANETs) appear where the topology keeps changing

- Dynamic SPRP (DSPRP)in MANETs:

- Find a series of shortest paths in a series of highly-related network topologies

- We model the network dynamics as follows:

- For each change, a number of nodes are randomly selected to sleep or wake up based on their current status
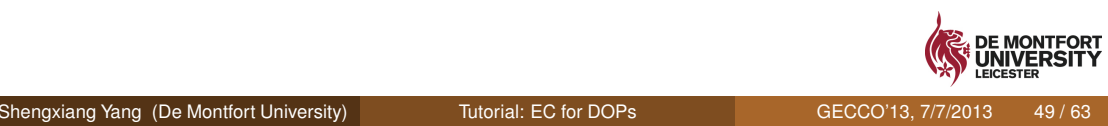

Tutorial: EC for DOP
Case Study: Dynamic Routing in MANETs - 2 எecco.

- A specialized GA for the DSPRP:

- Path-oriented encoding

- Tournament selection

- Path-oriented crossover and mutation with repair

- Enhancements: Immigrants and memory approaches

- Experimental results:

- Both immigrants and memory enhance GA's performance for the DSPRP in MANETs.

- Immigrants schemes show their power in acyclic environments

- Memory related schemes work well in cyclic environments

- More details:

- S. Yang, H. Cheng, \& F. Wang (2010). IEEE Trans SMC Part C: Appl. \& Rev., 40(1): 52-63

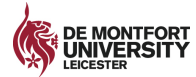

Case Study: Dynamic Vehicle Routing -1

- The basic Vehicle Routing Problem (VRP):

- A number of vehicles with a fixed capacity need to satisfy the demand of all customers, starting from and finishing to the depot

- Dynamic extensions of VRP that model real-world scenarios:

- Dynamic demands

- Traffic factors

- Dynamic test cases can be generated using the DBGP generator (Mavrovouniotis et al., 2012)

Case Study: Dynamic Vehicle Routing -2

- ACO algorithms with immigrants schemes are used to address the dynamic VRP with traffic factors

- Each ant constructs a solution that contains all the routes of the vehicles

- Diversity is maintained using immigrant ants

- Experimental results:

- ACO with elitism-based immigrants outperforms other ACO algorithms - ACO with random immigrants is outperformed by other ACO algorithms

- Usually, ACO with guided diversity performs well for DOPs

- More details:

- M. Mavrovouniotis \& S. Yang (2012a). EvoApplications'12, LNCS 7248, pp. $519-528$

- M. Mavrovouniotis \& S. Yang (2012b). CEC'12 
- Dynamic TSP

- 144 Chinese cities, 1 geo-stationary saterllite, and 3 mobile satellites

- Find the path that cycles each city and satellite once with the minimum length over time

- Solver: A GA with memory and other schemes

- More details:

- C. Li, M. Yang, \& L. Kang (2006). SEAL'O6, LNCS 4247, pp. 236-243
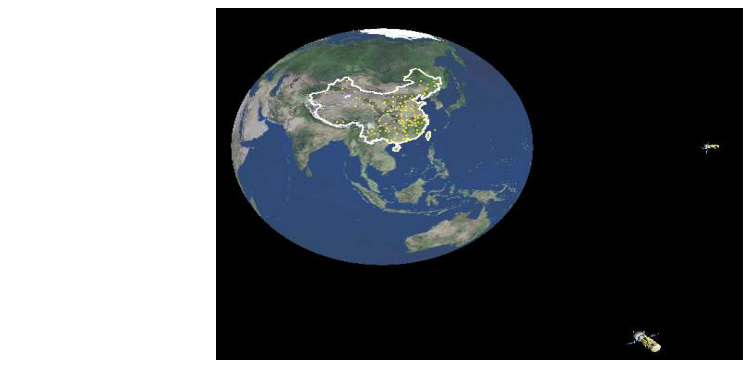

\section{Theoretical Development

- So far, mainly empirical studies

- Theoretical analysis has just appeared

- Runtime analysis:

- Stanhope \& Daida (1999) first analyzed a (1+1) EA on the dynamic bit matching problem (DBMP)

- Droste (2002) analyzed the first hitting time of a (1+1) ES on the DBMP

- Rohlfshagen et al. (2010) analyzed how the magnitude and speed of change may affect the performance of the $(1+1)$ EA on two functions constructed from the XOR DOP generator

- Analysis of dynamic fitness landscape:

- Branke et al. (2005) analyzed the changes of fitness landscape due to changes of the underlying problem instance

- Richter (2010) analyzed the properties of spatio-temporal fitness landscapes constructed from Coupled Map Lattices (CML)

- Tinos and Yang (2010) analyzed the properties of the XOR DOP generator based on the dynamical system approach of the GA

\section{Challenging Issues}

- So far, mainly dynamic single-objective optimization

- Dynamic multi-objective optimization problems (DMOPs): even more challenging

- A few studies have addressed EC for DMOPs

- Farina et al. (2004) classified DMOPs based on the changes on the Pareto optimal solutions

- Goh \& Tan (2009) proposed a competitive-cooperative coevolutionary algorithm for DMOPs

- Zeng et al. (2006) proposed a dynamic orthogonal multi-objective EA (DOMOEA) to solve a DMOP with continuous decision variables

- Zhang \& Qian (2011) proposed an artificial immune system to solve constrained DMOPs

- Detecting changes:

- Most studies assume that changes are easy to detect or visible to an algorithm whenever occurred

- In fact, changes are difficult to detect for many DOPs

- Understanding the characteristics of DOPs:

- What characteristics make DOPs easy or difficult?

- The work has started, but needs much more effort

- Analysing the behaviour of EAs for DOPs:

- Requiring more theoretical analysis tools

- Addressing more challenging DOPs and EC methods

- Big question: Which EC methods for what DOPs?

- Real world applications:

- How to model real-world DOPs? 


\section{Future Work}

- The domain has attracted a growing interest recently

- But, far from well-studied

- New approaches needed: esp. hybrid approaches

- Theoretical analysis: greatly needed

- EC for DMOPs: deserves much more effort

- Real world applications: also greatly needed

- Fields: logistics, transport, MANETs, data streams, social networks, ...
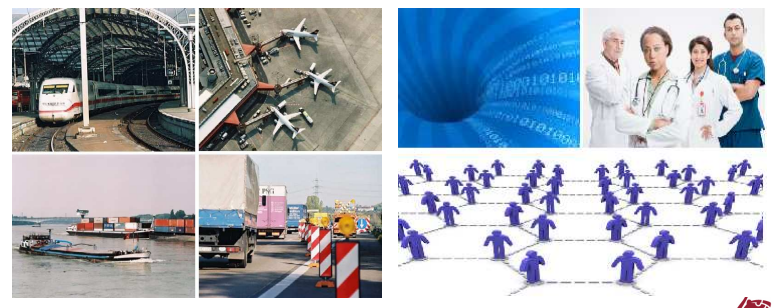

(4:)
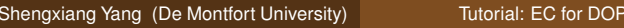

\section{Acknowledgements}

- Two EPSRC funded projects on EC for DOPs

- "EAs for DOPs: Design, Analysis and Applications"

- Linked project among Brunel Univ. (Univ. of Leicester before 7/2010), Univ. of Birmingham, BT, and Honda

- Funding/Duration: over £600K / 3.5 years (1/2008-7/2011)

- http://www.cs.le.ac.uk/projects/EADOP/

- "EC for Dynamic Optimisation in Network Environments"

- Linked project among DMU, Univ. of Birmingham, RSSB, and Network Rail

- Funding/Duration: £1M / 4 years (2/2013-2/2017)

- http://www.cci.dmu.ac.uk/research-grants/

- Research team members:

- Research Fellows: Dr. Hui Cheng, Dr. Crina Grosan, Dr. Changhe Li, Dr. Michalis Mavrovouniotis

- PhD students: Changhe Li, Michalis Mavrovouniotis, Lili Liu, Hongfeng Wang, Yang Yan

- Research cooperators:

- Prof. Xin Yao, Prof. Juergen Branke, Dr. Renato Tinos, Dr. Hendrik Richter, Dr. Trung Thanh Nguyen, etc. rsity)

\section{GECCO'13, 7}

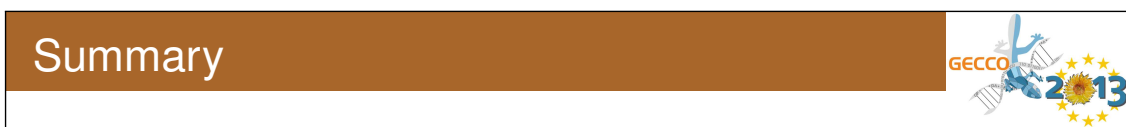

- EC for DOPs: challenging but important

- The domain is still young and active:

- More challenges to be taken regarding approaches, theory, and applications

- More young researchers are greatly welcome!

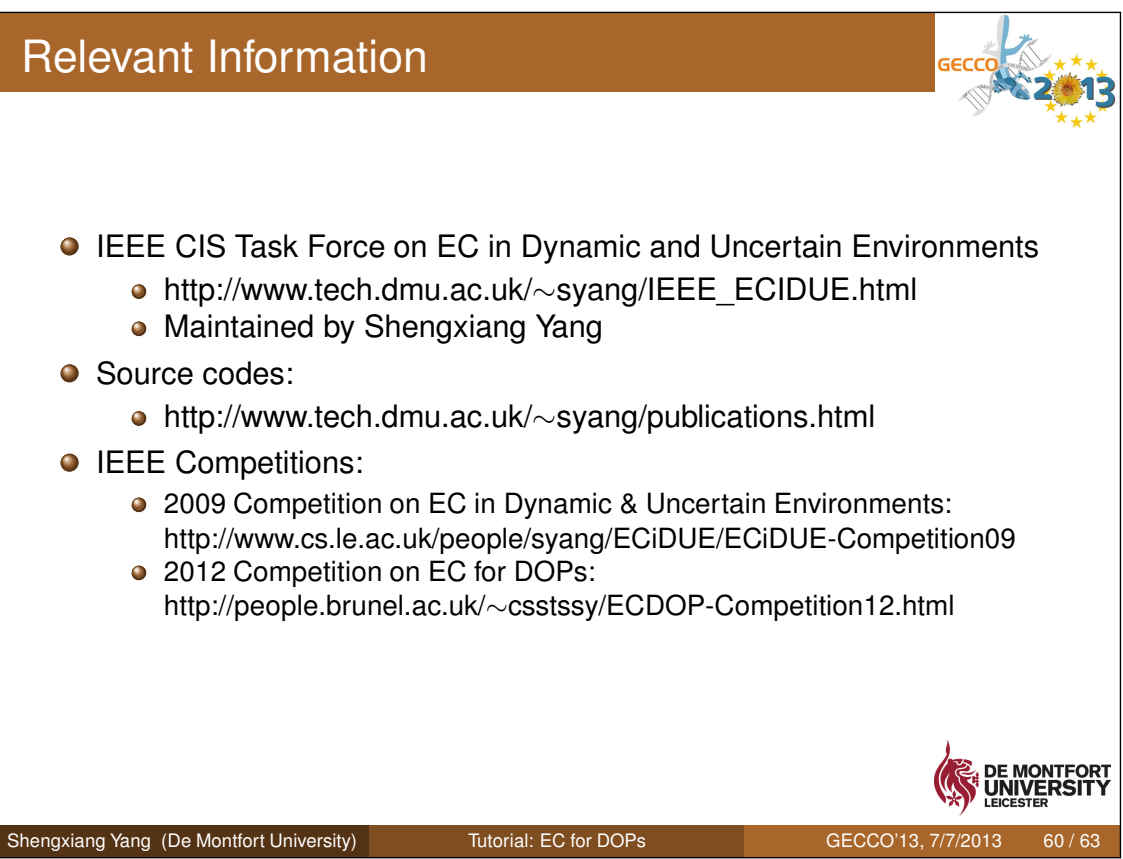




\section{References -1}

(1) J. Branke (1999). Memory enhanced evolutionary algorithms for changing optimization problems. CEC'99, pp. 1875-1882

J. Branke (2002). Evolutionary Optimization in Dynamic Environments. Kluwer Academic Publishers

3 J. Branke, E. Salihoglu \& S. Uyar (2005). Towards an analysis of dynamic environments. GECCO'05, pp. 1433-1439

4) H.G. Cobb \& J.J. Grefenstette (1993). Genetic algorithms for tracking changing environments. Proc. ICGA, pp. 523-530

C. Cruz, J. Gonzanlez, \& D. Pelta (2011). Optimization in dynamic environments: A survey on problems, methods and measures. Soft Comput., 15: 1427-1448

S. Droste (2002) Analysis of the $(1+1)$ ea for a dynamically changing onemax-variant. CEC'02, pp. 55-60

(7) M. Farina, K. Deb, \& P. Amato (2004). Dynamic multiobjective optimization problems: test cases, approximations, and applications. IEEE Trans. Evol. Comput., 8(5): 425-442

C. Goh \& K.C. Tan (2009). A competitive-cooperative coevolutionary paradigm for dynamic multiobjective optimization. IEEE Trans. Evol. Comput., 13(1): 103-127

Y. Jin \& J. Branke (2005). Evolutionary optimization in uncertain environments-A survey. IEEE Trans. Evol. Comput., 9(3): 303-317

(10 R.W. Morrison (2004). Designing Evolutionary Algorithms for Dynamic Environments. Springer

(1) E.H.J. Lewis \& G. Ritchie (1998). A comparison of dominance mechanisms and simple mutation on non-stationary problems. PPSN V, pp. 139-148.

R.W. Morrison \& K.A. De Jong (1999). A test problem generator for non-stationary environments. CEC'99, pp. 2047-2053

\section{References -3}

(24) S. Yang, Y. Jiang, \& T.T. Nguyen (2013). Metaheuristics for dynamic combinatorial optimization problems. IMA J of Management Math., in press

25 S. Yang, Y.-S. Ong \& Y. Jin (2007). Evolutionary Computation in Dynamic and Uncertain Environments. Springer

$26 \mathrm{~S}$. Yang \& H. Richter (2009). Hyper-learning for population-based incremental learning in dynamic environments. CEC'09, pp. 682-689

27. S. Yang \& R. Tinos (2008). Hyper-selection in dynamic environments. CEC'08, pp. 3185-3192

28 S. Yang \& X. Yao (2005). Experimental study on population-based incremental learning algorithms for dynamic optimization problems. Soft Comput., 9: 815-834

29) S. Yang \& X. Yao (2008). Population-based incremental learning with associative memory for dynamic environments. IEEE Trans Evol Comput, 12: 542-561

$30 \mathrm{~S}$. Yang \& X. Yao (2013). Evolutionary Computation for Dynamic Optimization Problems. Springer

(31) K. Weicker (2003). Evolutionary Algorithms and Dynamic Optimization Problems. Der Andere Verlag

(32) S. Zeng et al. (2006). A dynamic multi-objective evolutionary algorithm based on an orthogonal design. CEC'06, pp. 573-580

(33 Z. Zhang \& S. Qian (2011). Artificial immune system in dynamic environments solving time-varying non-linear constrained multi-objective problems. Soft Comput., 15(7): 1333-1349

\section{References -2}

(3) K.P. Ng \& K.C. Wong (1995). A new diploid scheme and dominance change mechanism for non-stationary function optimisation. ICGA 6, pp 159-166

T.T. Nguyen, S. Yang, \& J. Branke (2012). Evolutionary dynamic optimization: A survey of the state of the art. Swarm \& Evol. Comput., 6: 1-24

(15) F. Oppacher \& M. Wineberg (1999). The Shifting balance genetic algorithm: Improving the GA in a dynamic environment. GECCO'99, vol. 1, pp. 504-510

(16) H. Richter (2010). Evolutionary optimization and dynamic fitness landscapes: From reaction-diffusion systems to chaotic $\mathrm{cml}$. Evolutionary Algorithms and Chaotic Systems, Springer, pp. 409-446.

(1) P. Rohlfshagen, P.K. Lehre, \& X. Yao (2009). Dynamic evolutionary optimisation: An analysis of frequency and magnitude of change. GECCO'09, pp. 1713-1720

S.A. Stanhope \& J.M. Daida (1999). (1+1) genetic algorithm fitness dynamics in a changing environments. CEC'99, vol. 3, pp. 1851-1858

R. Tinos \& S. Yang (2010) An analysis of the XOR dynamic problem generator based on the dynamical system. PPSN XI, LNCS 6238, Part I, pp. 274-283

A. Simões \& E. Costa (2009). Improving prediction in evolutionary algorithms for dynamic environments. GECCO'09, pp. 875-882

K. Trojanowski \& Z. Michalewicz (1999). Searching for optima in non-stationary environments. CEC'99, vol. 3, pp. 1843-1850

A.S. Uyar \& A.E. Harmanci (2005). A new population based adaptive domination change mechanism for diploid genetic algorithms in dynamic environments. Soft Comput., 9: 803-814

3 S. Yang (2003). Non-stationary problem optimization using the primal-dual 